\title{
Role of Inhibition for Temporal and Spatial Odor Representation in Olfactory Output Neurons: A Calcium Imaging Study
}

\author{
SILKE SACHSE AND C. GIOVANNI GALIZIA \\ Institut für Biologie-Neurobiologie, Freie Universität Berlin, D-14195 Berlin, Germany
}

Received 23 April 2001; accepted in final form 18 October 2001

Sachse, Silke and C. Giovanni Galizia. Role of inhibition for temporal and spatial odor representation in olfactory output neurons: a calcium imaging study. J Neurophysiol 87: 1106-1117, 2002; $10.1152 /$ jn.00325.2001. The primary olfactory brain center, the antennal lobe (AL) in insects or the olfactory bulb in vertebrates, is a notable example of a neural network for sensory processing. While physiological properties of the input, the olfactory receptor neurons, have become clearer, the operation of the network itself remains cryptic. Therefore we measured spatio-temporal odor-response patterns in the output neurons of the olfactory glomeruli using optical imaging in the honeybee Apis mellifera. We mapped these responses to identified glomeruli, which are the structural and functional units of the AL. Each odor evoked a complex spatio-temporal activity pattern of excited and inhibited glomeruli. These properties were odor- and glomerulus-specific and were conserved across individuals. We compared the spatial pattern of excited glomeruli to previously published signals, which derived mainly from the receptor neurons, and found that they appeared more confined, showing that inhibitory connections enhance the contrast between glomeruli in the AL. To investigate the underlying mechanisms, we applied GABA and the GABA-receptor antagonist picrotoxin (PTX). The results show the presence of two separate inhibitory networks: one is GABAergic and modulates overall AL activity, the other is PTX-insensitive and glomerulus-specific. Inhibitory connections of the latter network selectively inhibit glomeruli with overlapping response profiles, in a way akin to "lateral" inhibition in other sensory systems. Selectively inhibited glomeruli need not be spatial neighbors. The net result is a globally modulated, contrast-enhanced and predictable representation of odors in the olfactory output neurons.

\section{N T R O D U C T I O N}

In recent years, research in olfaction has considerably enriched understanding of how the brain works. Olfactory receptor genes have been cloned in vertebrates (reviewed by Mombaerts 1999) and in Drosophila (Clyne et al. 1999; Vosshall et al. 1999), and the projection of afferent axons to the olfactory bulb and its insect analog, the antennal lobe (AL), has been visualized (Gao et al. 2000; Mombaerts et al. 1996; Vosshall et al. 2000), showing a strict topology of its functional processing units, the olfactory glomeruli. Odor-specific spatio-temporal activity patterns of these olfactory glomeruli have been shown in vertebrates (Cinelli et al. 1995; Friedrich and Korsching 1997, 1998; Kauer et al. 1987; Lam et al. 2000; Meister and Bonhoeffer 2001; Rubin and Katz 1999; Uchida et al. 2000) as

Address for reprint requests: S. Sachse, Institut für Biologie-Neurobiologie, Königin-Luise Str. 28-30, 14195 Berlin, Germany (E-mail: silsis@ zedat.fu-berlin.de). well as in invertebrates (Galizia et al. 1999b, 2000; Joerges et al. 1997; Sachse et al. 1999) and indicated that odors are represented as combinatorial activity patterns of glomeruli. The olfactory bulb and the AL are stereotypical examples of neural networks that process sensory information, where an enormous input via afferent fibers is transformed into an output consisting of a comparatively small number of neurons (Hildebrand and Shepherd 1997; Schild 1988). These networks are ideal models for computational analyses of biological neural networks. Still, our understanding of the olfactory code is limited, and this limitation is also due to our inability to follow the physiological responses along their path when being processed by its neural components. We have now succeeded in filling one gap in the analysis of the olfactory code using calcium imaging in the honeybee. We measured the spatiotemporal response patterns of the processed information, i.e., of the output neurons of the olfactory glomeruli, to investigate how the AL shapes the olfactory code.

In the honeybee Apis mellifera, 60,000 olfactory receptor neurons (RNs) are housed in antenna sensilla (Esslen and Kaissling 1976) and convey input signals to the AL through four tracts (T1-T4) (Suzuki 1975). The sensory axons converge on two categories of AL neurons, namely 4000 local interneurons (LNs) (Witthöft 1967), which exclusively branch within the AL, and about 800 projection neurons (PNs) (Bicker et al. 1993), which represent the AL output. The synaptic contacts between RNs, LNs, and PNs are localized in areas of high-synaptic density, the AL glomeruli (Gascuel and Masson 1991). Each glomerulus represents an identifiable morphological and functional unit, of which there are about 160 arranged in a single layer around the AL (Arnold et al. 1985; Flanagan and Mercer 1989b). Glomeruli can be individually identified on the basis of their shape and relative position and are named according to the antennal tract which innervates them (T1 to T4), followed by a number, e.g., T1-17 or T1-33 (Flanagan and Mercer 1989a; Galizia et al. 1999a). The output information from the $\mathrm{AL}$ is relayed to the lateral protocerebrum and the mushroom bodies via three different antenno-cerebral tracts (ACT; Fig. 1, $A$ and $B$ ) (Mobbs 1982). The small medio-lateral ACT (ml-ACT) contains pluriglomerular cells. The lateral and median ACTs (1- and m-ACTs) contain axons of uniglomerular PNs (Abel et al. 2001), innervating glomeruli of T1 or T2 and T3, respectively (Abel et al. 2001; Bicker et al. 1993) (Fig. 1,

The costs of publication of this article were defrayed in part by the payment of page charges. The article must therefore be hereby marked "advertisement" in accordance with 18 U.S.C. Section 1734 solely to indicate this fact. 


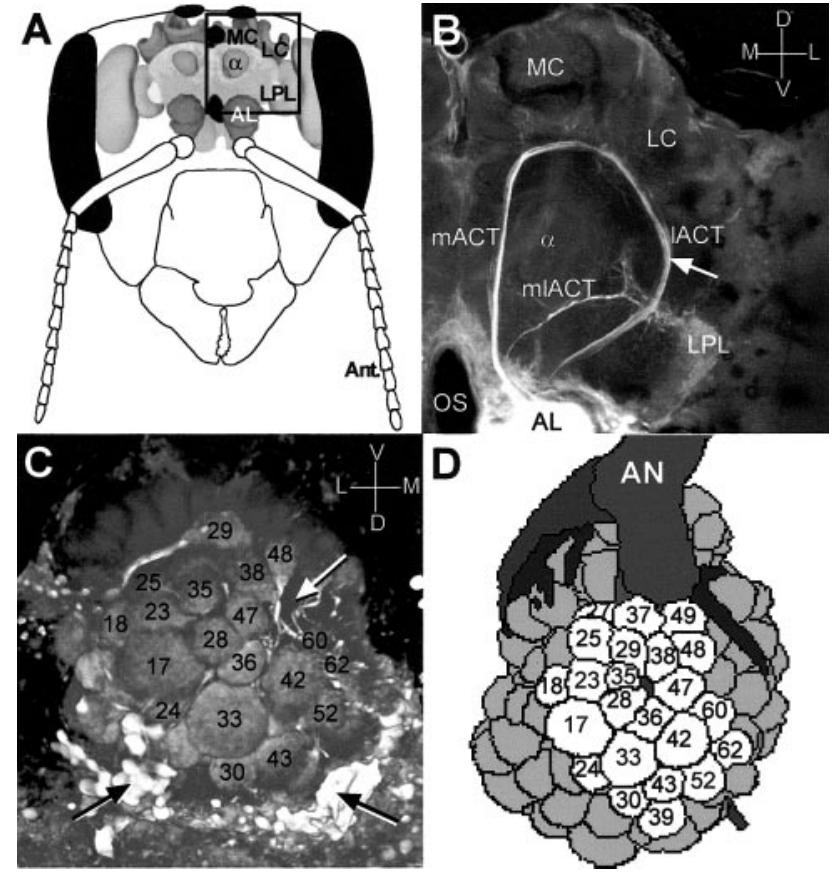

FIG. 1. The olfactory output neurons of the honeybee antennal lobe (AL). $A$ : schematic view of the honeybee brain. The olfactory pathway leads from the antenna (Ant.) to the first olfactory neuropil, the AL. The PNs send their axons to higher processing centers: the lateral protocerebrum (LPL) and the medial and lateral mushroom body calyx (MC and LC). $\alpha$ : $\alpha$-lobe of the mushroom body. $B$ : confocal section of a honeybee brain showing the area marked by a rectangle in $A$. The output neurons were anterogradely stained by applying tetramethylrhodamine-dextran to the AL. The projection neurons (PNs) leave the AL via the median, the lateral, and the medio-lateral antenno-cerebralis tracts $(\mathrm{m}-, 1-$, and $\mathrm{ml-ACT})$. For retrograde 1-ACT staining, we aimed at the position marked by the white arrow. OS: esophagus. $C$ : confocal section of the $\mathrm{AL}$ in another animal, in which the 1-ACT neurons were retrogradely fluorescently stained by inserting tetramethylrhodamine-dextran at the same position as has been used for the calcium-sensitive dye (white arrow in $B$ ). Only glomeruli of the T1 tract were stained (some of them are not in the focal plane and therefore not visible, e.g., glomeruli 27, 37, 49), which are labeled with their identities corresponding to the digital AL atlas (Galizia et al. 1999a). Glomeruli of tract 3 are not stained (the white arrow indicates the position where glomerulus T3-45 would appear), because they are innervated by the m-ACT neurons. The black arrows mark the soma clusters of the 1-ACT neurons. Note that this image is turned upside down as compared with $B$ and corresponds to the AL orientation in all other figures. $D$ : schematic view of the $\mathrm{AL}$ with the 23 most frequently recognized glomeruli labeled with their identities (AN: antennal nerve).

$C$ and $D$ ). Each glomerulus is innervated by about three to five uniglomerular PNs of the 1- or m-ACT. Since only glomeruli on the exposed surface of the AL are accessible for optical imaging and since these are T1-glomeruli, we selectively backfilled the 1-ACT with the calcium-sensitive dye fura-dextran and optically recorded its glomerular response properties. We measured the spatio-temporal representation of the alcohols 1-hexanol, 1-octanol, 1-nonanol, and the alarm pheromone isoamylacetate, and evaluated the activity patterns at the level of morphologically identified glomeruli using a digital AL atlas (Galizia et al. 1999a). In previous imaging studies, where we bath-applied the calcium-sensitive dye and thus labeled all AL cells, a homologous series of aliphatic alcohols evoked overlapping activity patterns, which were often restricted to neighboring glomeruli (Sachse et al. 1999). To ensure a specific recognition of chemically similar odors, which is realized for the honeybee and has been shown with behavioral experiments (Laska et al. 1999), the activity patterns have to be sharpened in the neural network. This could possibly be accomplished by a narrowing of the glomerular response profiles through a lateral inhibition of the PNs, which is shown for vertebrates (Margrie et al. 2001; Yokoi et al. 1995). Here, we examine such processing properties of the AL by measuring the processed odor representation and comparing it with the input information. Application of GABA and its receptor antagonist picrotoxin (PTX) to the AL provided us with information about inhibitory mechanisms, leading to a proposal about the wiring of the AL network.

\section{METHODS}

\section{Animal preparation and staining process}

Adult worker honeybees were caught from various hives in the morning, fixed in a Plexiglas stage using dental wax, and fed with sucrose solution. A small window was cut into the cuticle and glands and trachea were carefully removed. A glass electrode was coated with crystals of fura-dextran (potassium salt, $3000 \mathrm{MW}$, Molecular Probes, Eugene, OR), dissolved in $2 \%$ bovine serum albumin solution, and inserted into the left deutocerebrum lateral to the $\alpha$-lobe, aiming for the 1-ACT. A similar dye application method was shown to be effective for calcium-green-dextran (Delaney et al. 2001; Gelperin and Flores 1997). The brain was rinsed with Ringer solution (130 mM $\mathrm{NaCl}, 6 \mathrm{mM} \mathrm{KCl}, 4 \mathrm{mM} \mathrm{MgCl}$, $5 \mathrm{mM} \mathrm{CaCl}_{2}, 160 \mathrm{mM}$ sucrose, 25 $\mathrm{mM}$ glucose, $10 \mathrm{mM}$ HEPES, pH 6.7, 500 mosmol; all chemicals from Sigma) to remove extracellular dye. After $3 \mathrm{~h}$ of staining, the antennae were immobilized with two-component silicon (Kwik-Sil, WPI) and the abdomen was cut. The preparation was covered with a coverslip and constantly superfused with Ringer during measurements ( $1 \mathrm{ml} / \mathrm{min})$. Clear signals were registered in 13 of 155 bees tested. This small yield indicates the selectivity of the backfill method: only when the electrode hit the l-ACT, the dye was transported to the AL within the PNs, and we could measure calcium responses in the glomeruli. Since Molecular Probes discontinued producing fura-dextran $3000 \mathrm{MW}$, control experiments with fura-dextran 10,000 MW were performed showing identical results. We also measured PN responses in one animal which was stained with calcium-green-1dextran (potassium salt, $3000 \mathrm{MW}$, Molecular Probes), confirming that the dynamic patterns of PNs were not dependent on the dye used.

In control experiments, we anterogradely stained the three ACTs by inserting the dye tetramethylrhodamine-dextran (3000 MW, Molecular Probes) into the AL (Fig. 1B). Furthermore, we locally applied tetramethylrhodamine-dextran at the same position as was used for the calcium-sensitive dye to retrogradely stain the 1-ACT neurons and their corresponding glomeruli (Fig. $1 C$ ). Both preparations were imaged using confocal microscopy.

\section{Calcium measurements of $P N$ responses}

Imaging was done using a T.I.L.L. Photonics imaging system (Germany). Monochromatic excitation light alternated between 340 and $380 \mathrm{~nm}$, dicroic: $410 \mathrm{~nm}$, emission: LP $440 \mathrm{~nm}$. Measurements were done with an upright Axioskop microscope (Zeiss), using a Leica long distance $20 \times$ air objective (numeric aperture $=0.6$ ). Pixel image size was $2.4 \times 2.4 \mu \mathrm{m}$, obtained by $2 \times 2$ binning on chip. For each measurement, a series of either 60 or 90 double frames were taken with frequencies of $6 \mathrm{~Hz}$. Light was turned off between frames. Interstimulus interval was $40 \mathrm{~s}$, i.e., the animal was not exposed to light for about $30 \mathrm{~s}$ between measurements.

Odors were delivered to the antennae using a custom-made and computer-controlled olfactometer by switching from a constant air 
stream to an odor stream to eliminate mechanical stimulation (Galizia et al. 1997). Stimulus duration was 2 s. For each odor, $6 \mu \mathrm{l}$ of the odorant dissolved in mineral oil was applied on a filter paper $\left(1 \mathrm{~cm}^{2}\right)$ in a plastic syringe. Dilution was adjusted to equal effective vapor pressure for the different odorants. The control stimulus was a syringe plus filter paper with mineral oil. Odors used were isoamylacetate, 1-hexanol, 1-octanol, and 1-nonanol (Sigma-Aldrich, Deisenhofen).

Solutions of GABA (Sigma) and PTX (Sigma) were dissolved in Ringer for final concentrations of $10 \mathrm{mM}$ for GABA and 1,10 , and $100 \mu \mathrm{M}$ for PTX. Solutions of GABA and PTX were bath-applied and reached the brain within $30 \mathrm{~s}$ after the perfusion switch. We measured 6 of the 13 successfully stained bees with a standardized protocol of the pharmacological treatment. The standardized protocol consisted at least of two measurements for each odor during each treatment. The whole protocol started with Ringer application. PTX was applied with increasing concentrations for $12 \mathrm{~min}$ each. The brain was then washed with Ringer until the PTX effects were no longer visible ( $\geq 45 \mathrm{~min}$ ). Then GABA was applied for $12 \mathrm{~min}$. The GABA effects could be completely washed out after about $3 \mathrm{~min}$. In control animals we bath-applied GABA without prior incubation of PTX, showing that the GABA effect was not influenced by the preceding PTX application.

\section{Data processing}

Calcium concentration data are shown as absolute changes of fluorescence ratio between 340 and $380 \mathrm{~nm}$ excitation light. More specifically, for each pixel the false-color-coded images give the signal increase between frames 5 and 16

$$
\Delta(F 340 / F 380)_{F C}=\left(\frac{F 340_{15-17}}{F 380_{15-17}}-\frac{F 340_{4-6}}{F 380_{4-6}}\right) \times 1000
$$

For the time traces, values are given as

$$
\Delta(F 340 / F 380)_{i}=\left(\frac{F 340_{i}}{F 380_{i}}-\frac{F 340_{3-5}}{F 380_{3-5}}\right) \times 1000
$$

In these formulas, $F x x x_{i}$ indicates the fluorescence intensity for excitation light of $x x x \mathrm{~nm}$ in frame $i, F x x x_{i-j}$ indicates the averaged fluorescence intensity from frame $i$ to frame $j$. Subtracting frames 3-5 from the time traces leads to these traces being all superimposed at a value of 0 shortly before stimulus onset. This is necessary because PNs revealed a high amount of spontaneous activity and each glomerulus had individual background fluorescence. Furthermore, the ratios were median-filtered for shot noise reduction (filter size 3 pixels in two spatial and one temporal dimension) and were corrected for lamp noise by subtracting the median value of each frame from it. Calculations were done in IDL (Research Systems, CO). Changes in $\Delta(F 340 / F 380)$ of Fura are approximately proportional to changes of the intracellular calcium concentration. Their absolute values, however, are influenced by several experimental parameters, such as background fluorescence, staining intensity, and light exposure time. Therefore these absolute values vary greatly between individuals and cannot be compared.

We attributed the signals to identified glomeruli in the following way. The glomerular structure was visible in the fura ratio images, allowing us to identify the glomeruli on the basis of their morphological borderlines using a digital atlas of the AL (Galizia et al. 1999a) (Figs. $1 D$ and $2 A$ ). This method has been described in detail (Galizia et al. 1999c; Sachse et al. 1999). Since not all glomeruli were visible or identifiable in all animals, the total count differs for different glomeruli (on average 19 glomeruli per animal). For time courses squares of $11 \times 11$ pixels (corresponding to $26.4 \mu \mathrm{M}$ side length and always well within the glomerulus chosen) were placed onto the center of a glomerulus, their values were averaged, and the courses were plotted against time (Figs. 2, 3, and 5).
For each animal, glomerulus, and odor, we calculated the response as the maximum during stimulus application. The median was taken to average repeated stimulations within one animal. All responses within the noise limit were clipped to 0 . Noise was estimated as the $\mathrm{SD}$ of the time courses between frames 4 and 11 (i.e., before stimulus) averaged over all measurements in one animal. To compare animals with different background fluorescence and thus different maximal activities, we set the strongest glomerular response of each animal at $100 \%$ and scaled the other responses accordingly. We then calculated the species' glomerular response (Fig. 4) by taking the median response of all animals. A calcium decrease during stimulus application was assigned to the category "negative response" (Fig. 4). To calculate the number of glomeruli that were spontaneously active (Fig. 5B) or that responded to nonanol (Fig. $5 C$ ), we counted each activity event above noise limit. The resting calcium levels (Fig. $5 D$ ) were calculated from the percental $F 340 / F 380$ changes before, during, and after the pharmacological treatment of all animals.

Statistical analyses of the data were performed using JMP 3.2.1, Statistical Discovery Software (SAS, Cary, NC). We calculated the correlation matrix (Fig. 4, $A$ and $B$ ) based on the species' glomerular responses. We included the same subset of glomeruli $(n=22)$ for both data sets. We used a two-tailed paired $t$-test to verify significant differences during the pharmacological treatment (Fig. 5, $B-D$ ).

\section{RES U L T S}

\section{Selective staining of olfactory output neurons}

To selectively stain the uniglomerular output neurons of the AL, we aimed at the nerve tract 1-ACT, which connects T1glomeruli with higher order brain centers. We locally applied the calcium-sensitive dye fura-dextran into the deutocerebrum lateral next to the $\alpha$-lobe (Fig. $1 B$, white arrow), a place where the PNs of the 1-ACT are the only neurons which project to the AL. Since the fura-dextran is membrane-impermeable, it is incorporated and transported only by cells at the application site. Therefore a staining of the other neurons in the AL (RNs, LNs, and PNs in the m- and ml-ACT tracts) can be excluded. In control experiments we inserted tetramethylrhodamine-dextran using the same procedure and visualized it with the confocal microscope. These stainings confirmed that there was either no staining in the AL (indicating that the tract was not labeled), or selective staining of T1-glomeruli (Fig. 1C), which are the glomeruli innervated by 1-ACT neurons (Abel et al. 2001; Bicker et al. 1993). Morphologically, a successful loading was apparent when the somata of the 1-ACT neurons were stained (Fig. 1C, black arrows); physiologically, successful loading was apparent by a fast, "flickering" form of spontaneous activity (see following text).

\section{Spontaneous activity in olfactory output neurons}

We found two different types of spontaneous activity: one consisted of very brief and small changes in calcium concentration, which in the individual time courses is not distinguishable from random noise. When observed as a movie of the AL, it was clear that the glomeruli were active as units in this noise, so that the entire AL appeared as if the individual glomeruli were continuously flickering (see example on http://www. neurobiologie.fu-berlin.de/honeybeeALatlas/PN_physiol.htm). This flickering was a clear indicator of successfully stained PNs even in the absence of an olfactory stimulus. Only animals where all visible T1-glomeruli were successfully stained were 
A

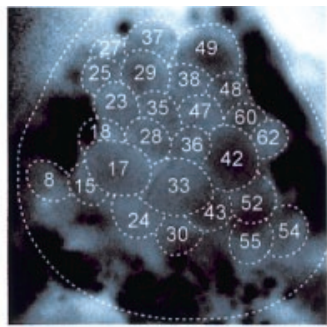

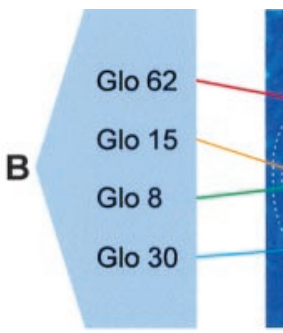
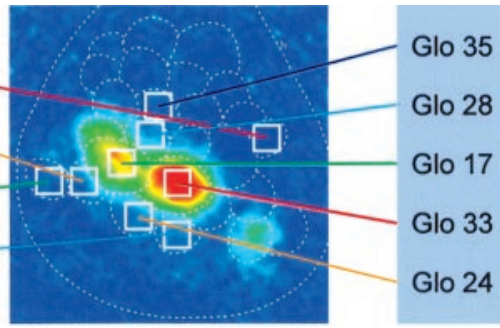

\section{C, D, E}
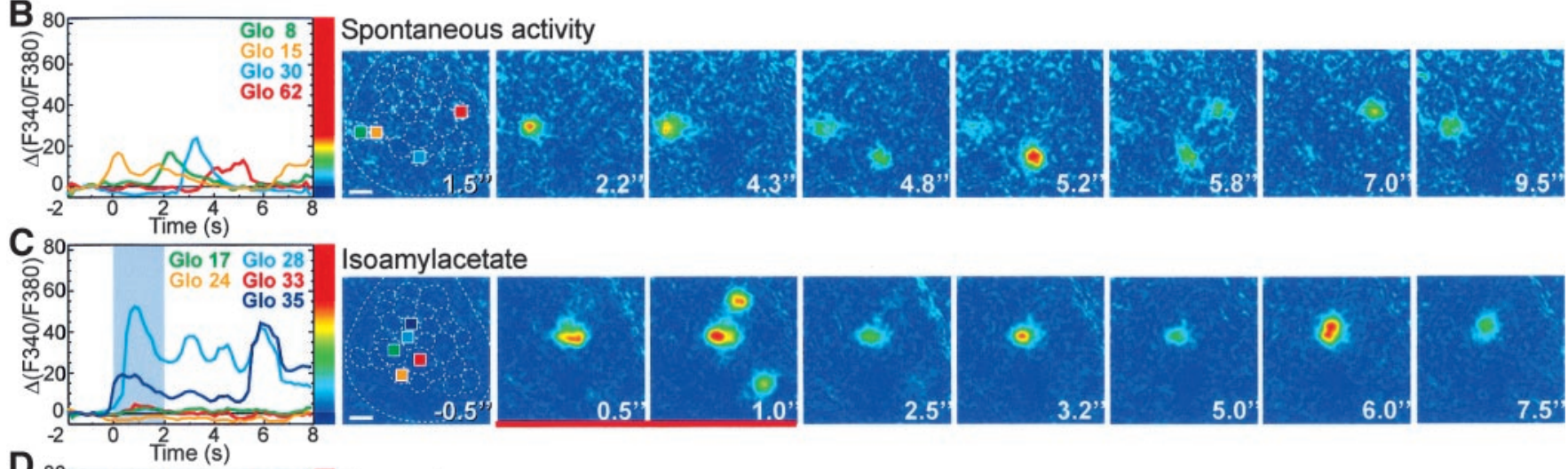

Isoamylacetate
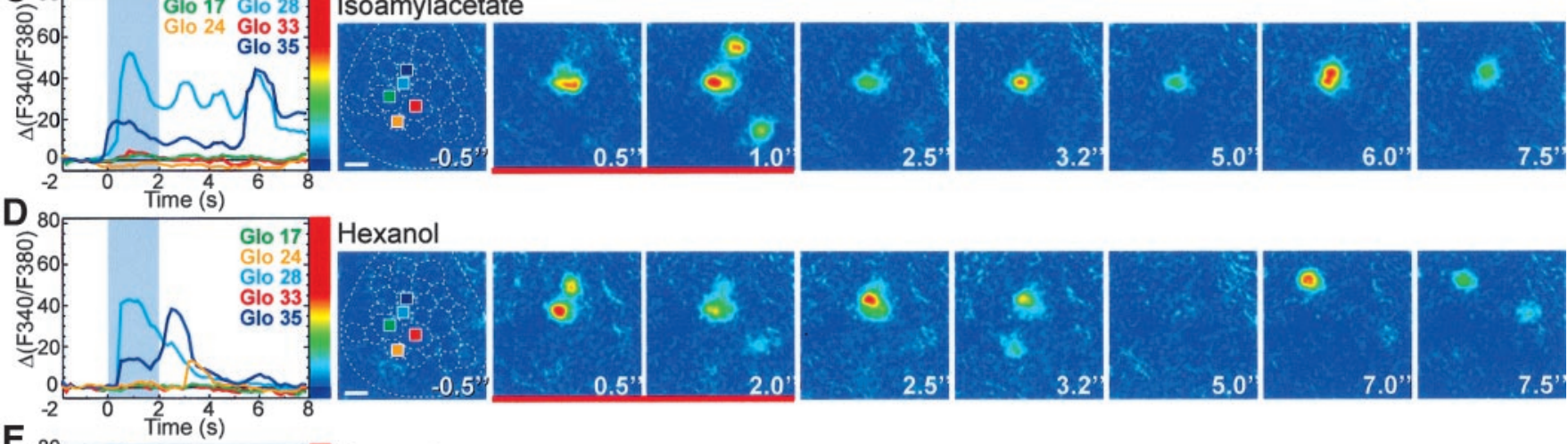

E

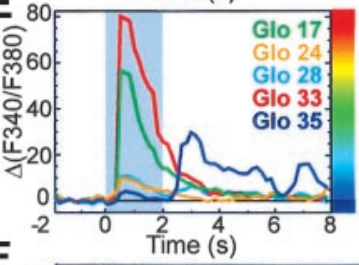

Nonanol

$\mathbf{F}$
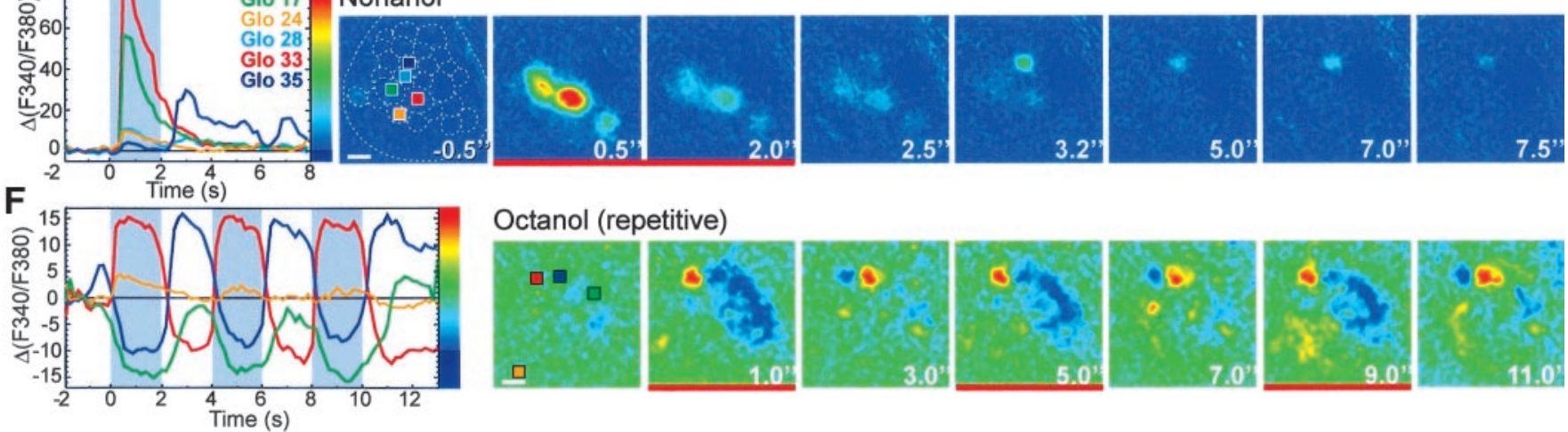

Octanol (repetitive)
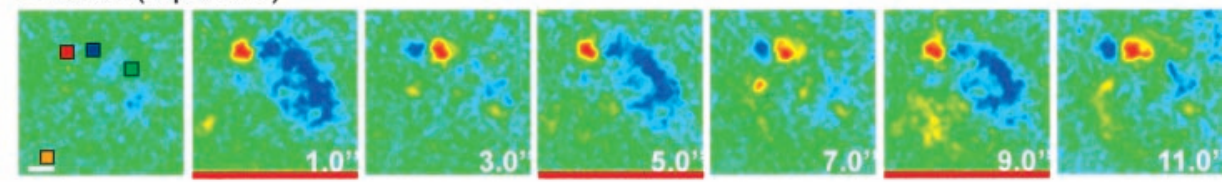

FIG. 2. Glomerular responses of selectively stained PNs in the honeybee AL. All sequences can be seen as movies at http://www.neurobiologie.fu-berlin.de/honeybeeALatlas/PN_physiol.htm. A, left: morphological view of the AL, where the glomerular structure and the border of the AL have been reconstructed. The single glomeruli have been identified using the digital AL atlas. Right: false-color-coded spatial response pattern to nonanol. Red indicates strongest response; blue indicates the weakest response. The reconstructed glomerular map is superimposed onto the response pattern. White boxes mark positions of glomeruli evaluated in $B$ (left) or $C-E$ (right). $B-E$ : time traces of identified glomeruli (left) and selected false-color-coded frames (right) from a movie of $10 \mathrm{~s}$ (same animal as in $A$ ). The colors of the time traces correspond to identified glomeruli, whose positions are marked by colored boxes in the first frame. The numbers at the bottom in each frame show time after measurement onset (in s). Odor-stimulus is marked by a shaded area in the time courses and a red bar in the frames ( $B$ : no stimulus, $C$ : isoamylacetate, $D$ : hexanol, $E$ : nonanol). Note the different scales of the false-color coding. $F$ : time traces (left) and selected frames from a movie (right) during repetitive stimulation with octanol (different animal from $A-E$ ). The stimulus is given 3 times for $2 \mathrm{~s}$ with a frequency of $0.25 \mathrm{~Hz}$. Note the different false-color scaling in comparison to the other frame series (here, green represents no response and blue represents a calcium decrease). The absolute values differ between animals (see METHODS). All scale bars correspond to $50 \mu \mathrm{m}$.

included in the evaluation of the data. In addition, PNs showed a slow spontaneous activity, with individual events clearly above noise levels (Fig. 2B). Again, the active units corresponded to individual glomeruli, which in most cases could be identified morphologically using a digital AL atlas (Galizia et al. 1999a) (Figs. $1 D$ and 2A). On average, seven activity events of individual glomeruli were visible during a measurement of $10 \mathrm{~s}$ in a field of view comprising about 19 glomeruli. This form of spontaneous activity was reminiscent of what has also been found in electrophysiological recordings of PNs of honey- 
bees, moths, and lobsters (Abel 1997; Kanzaki et al. 1989; Sun et al. 1993; Wachowiak and Ache 1998), consisting of irregular background spontaneous activity with alternating spike bursts (Abel 1997; Kanzaki et al. 1989).

\section{Odor response properties of olfactory output neurons}

Olfactory stimulation with the odors isoamylacetate, 1-hexanol, 1-nonanol, and 1-octanol led to specific and complex spatio-temporal activity patterns (Fig. 2, $C-F$ ), which were above spontaneous activity level. These patterns always consisted of a group of glomeruli, increasing in intracellular calcium concentration at stimulus onset, and another group showing an increase at stimulus offset. For most odors and animals we also observed glomeruli that decreased their calcium concentration either at or after stimulation with an odor. For example, in the animal shown in Fig. $2 C$, application of isoamylacetate evoked a sequenced activation of five glomeruli: within $500 \mathrm{~ms}$ after stimulus onset two glomeruli showed a strong calcium increase (glomerulus 28: cyan line, glomerulus 36: not shown as trace in Fig. 2C). Glomerulus 28 revealed repeated activity peaks until the end of the measurement. At $1000 \mathrm{~ms}$ after stimulus onset another two glomeruli joined the pattern (glomeruli 49 and 55: not shown as traces in Fig. $2 C$ ), whose activity ended after stimulus offset. Glomerulus 35 (blue line in Fig. 2C) showed a calcium increase shortly before and during the stimulus application. While this increase was consistent across measurements and animals, its appearance before stimulus in the measurement shown was unique and indicates a spontaneous component. Glomerular response patterns differed between odors: hexanol elicited a consistent strong but short lasting response in glomeruli 28 (cyan line in Fig. 2D) and 38 (not shown) shortly after stimulus onset. After $1.8 \mathrm{~s}$ (i.e., still within the stimulus) glomerulus 35 (blue line) increased its response, which became maximal at stimulus offset. This off response was unique for this measurement and thus probably spontaneous. Glomerulus 24 (yellow line) consistently showed an excitation $1 \mathrm{~s}$ after stimulus offset, which we therefore classed as an "OFF" response; conversely, the two glomeruli active at $7.5 \mathrm{~s}$ after stimulus onset were not reliably active for repeated measurements, suggesting that they were spontaneously active. Nonanol elicited a diagonal pattern of two glomeruli (17 and 33, Fig. 2E) at stimulus onset, an ofF response in glomerulus 35 , and a weak excitation in glomerulus 24. Thus in the two glomeruli 35 and 24 the chemically closely related odors hexanol and nonanol elicited opposing responses. We tested the mutual relationship of ON- and OFFresponses by repetitively stimulating with an odor at a frequency of $0.25 \mathrm{~Hz}$ (Fig. $2 F$, different animal from Fig. 2, $A-E$ ). Interestingly, two neighboring glomeruli showed opposite responses which were directly correlated to stimulus timing: the glomerulus marked in red was strongly activated during stimulus application and showed a calcium decrease at stimulus offset, whereas the glomerulus marked in blue revealed a calcium decrease during stimulus application and an activation between the stimuli. Lengthening the stimulus from 2 to $4 \mathrm{~s}$ led to $\mathrm{PN}$ responses lasting $\geq 4 \mathrm{~s}$ as well (data not shown).

\section{Comparison of the PN activity patterns between different individuals}

The spatial patterns of glomeruli strongly activated by isoamylacetate, hexanol, and nonanol were identical for all bees tested ( $n=7$, Fig. 3A). These were glomeruli 28 and 49
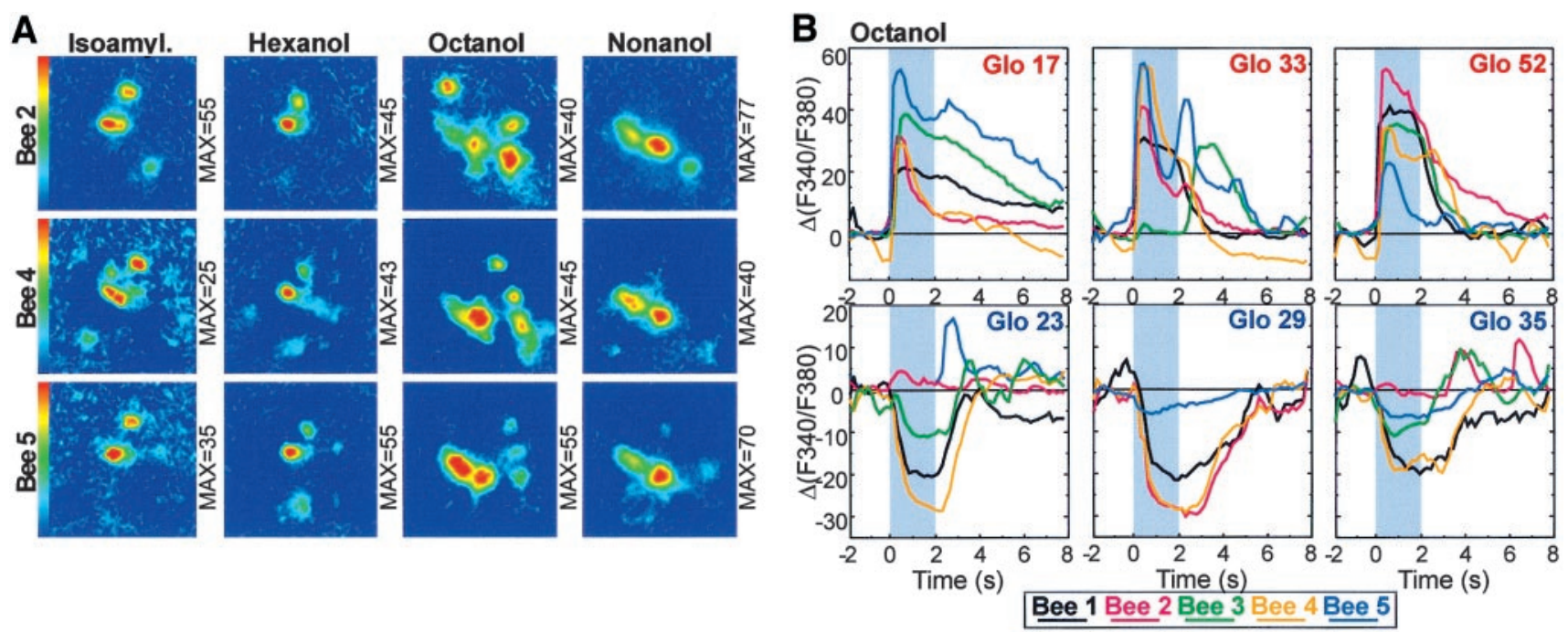

FIG. 3. Odor stimulation leads to glomerular patterns of calcium increase and decrease of PNs; these patterns are conserved across individuals. A: false-color-coded spatial response patterns of 3 different honeybees during stimulation with the odors isoamylacetate, hexanol, octanol, and nonanol. The maximal response intensity is given for each frame to the right. Calcium decreases are not visible, since the false-color scale was cut at 0 . In all three bees, isoamylacetate evoked a strong calcium increase in glomeruli 28 and 49, hexanol elicited a strong response in glomerulus 28 and an intermediate response in glomerulus 38 , and nonanol leads to a strong activation of glomerulus 33 and an intermediate of glomerulus 17 . The activity patterns of octanol appear more variable; while the identity of activated glomeruli is equal between the bees, their relative activity strengths differ. $B$ : time traces of 5 different animals (marked by different colors) for 6 identified glomeruli during stimulation with octanol. Octanol elicits a calcium increase in glomeruli 17 (5 of 5 animals), 33 (4 of 5), and 52 (5 of 5), and a decrease in glomeruli 23 (4 of 5), 29 (4 of 4 ), and 35 (5 of 5). Response amplitudes cannot be compared between individuals (see METHODS). Differences between individuals may reflect individual experiences. 
for isoamylacetate, 28 and 38 for hexanol, and 17 and 33 for nonanol. Interestingly, the response to octanol was more variable between individuals, which is due to the shift of the strongest response between the glomeruli 52 (bee 2), 33 (bee 4), or 17 and 33 (bee 5), respectively. We classified responses as positive (calcium increase) or negative (calcium decrease, due to intraglomerular inhibition; see following text), and we compared the stimulus-response polarity of individual glomeruli between different individuals (Fig. 3B). Negative responses revealed themselves either as a calcium-concentration decrease during the stimulus or as a rebound calcium increase after stimulus offset (OFF response) due to release from inhibition. Response polarity was strongly conserved between animals for all tested odors. For example, in all animals, octanol, which was the most variable odor in the tested odor set, elicited a positive response in glomeruli 17, 33 (only one exception), and 52 and a negative in glomeruli 23 (one exception), 29, and 35 (Fig. 3B). Positive or negative responses differed with the odor: for example, glomerulus 35 , which revealed an inhibitory response to nonanol and octanol (Figs. $2 E$ and $3 B$ ), showed an excitatory response during isoamylacetate stimulation (Fig. $2 C)$. Besides the stereotypical glomerular responses, there is some variability which mainly affects response intensity. Part of it is probably a genuine variability between animals and part is due to experimental parameters, such as differences in autofluorescence (see DiscUSSION).

Since response polarity is conserved among individuals, we calculated the average representation of odors as patterns of excited and inhibited glomeruli in a schematic AL (Fig. 4A). Positive response intensities are given in five categories, while negative responses have only one category. Interestingly, the excited and inhibited glomeruli are not necessarily neighbors. For octanol they appear almost as clusters in the AL, which are separated through nonresponding glomeruli, in the response to nonanol, they are also clustered, but without nonresponding glomeruli in between, while they are interspersed throughout the AL for hexanol (Fig. 4A).

\section{Comparison of the input and the output neurons in the $A L$}

In previously published experiments, using a bath-application of the calcium-sensitive dye, we studied the glomerular activity as a "compound" signal of all cell types in the AL (RNs, LNs, and PNs) (reviewed in Galizia and Menzel 2001) with a probable emphasis on RNs (Galizia et al. 1998) and found those activity patterns to also be conserved within the species (Galizia et al. 1999c; Sachse et al. 1999). To compare the compound responses with the odor responses reported in this paper, we replicate the compound responses to hexanol, octanol, and nonanol in Fig. $4 B$ (from Sachse et al. 1999) alongside the PN responses in Fig. 4A. A strong similarity is apparent between the spatial pattern of the output neuron responses and the compound signal. In both, the activity pattern of hexanol is dominated by glomerulus 28 and 38, octanol by glomeruli 17,33 , and 52 , and nonanol by the characteristic diagonal pattern (glomerulus 17 and 33; compare with Fig. $1 D$ for glomerular identity). However, most glomeruli, which are intermediately or weakly active in the compound signal, show no calcium increase in their PNs. For example glomerulus 17 showed a medium strong response to hexanol (green category),

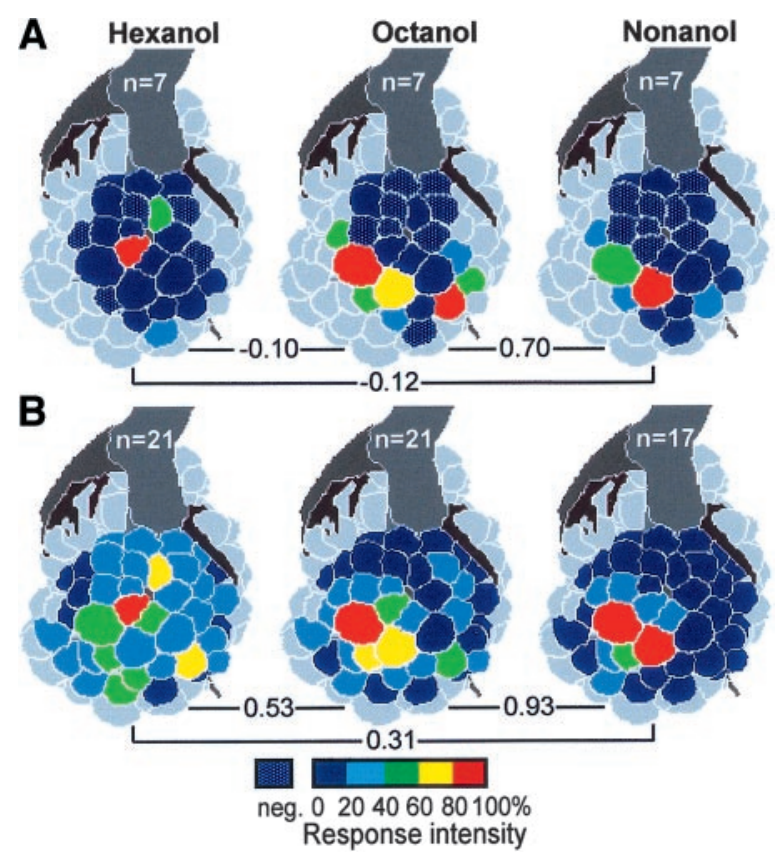

FIG. 4. Fewer glomeruli are involved in the PN activity patterns in comparison to a compound signal of all cell types [receptor neurons (RNs), local interneurons (LNs), and PNs]. A: glomerular activity patterns of selectively stained PNs responding to the odors hexanol, octanol, and nonanol, depicted with a schematic AL. The color code gives the relative response intensity in 5 bins (for calculation details see METHODS). Inhibitory responses are represented by an additional category. The correlation for each odor pair (calculated for the identical glomeruli subset of each) are represented at the bottom. B: spatial activity patterns of the previously obtained compound signal to the same odors as in $A$, measured with a bath-applied calcium-sensitive dye (categories have been attributed in an identical way; data from Sachse et al. 1999). No calcium decrease was seen. The correlation for each odor pair, as in $A$, is shown at the bottom. Comparison of $A$ with $B$ reveals a good match of strongly activated glomeruli. However, the PN patterns appear more selective due to the lack of weakly activated glomeruli.

but no PN response (dark blue category). The weak compound response of glomeruli 23, 35, and 48 during octanol stimulation results in negative responses of their PNs and the strong compound response of glomerulus 17 to nonanol (red category) is reduced to an intermediate PN response (green category). A correlation analysis of each odor pair shows that the glomerular activity patterns of the PNs are less correlated to each other than the patterns of the compound signal (Fig. 4, $A$ and $B$ ).

\section{Analyzing the inhibitory connections within the AL}

To analyze the influence of inhibitory neurons on the PN responses, we bath-applied GABA or the fast GABA receptor antagonist PTX. PTX has been shown to be effective in an insect AL preparation (MacLeod and Laurent 1996; MacLeod et al. 1998; Stopfer et al. 1997; Waldrop et al. 1987; Wehr and Laurent 1999); PTX reversibly blocked odor-induced inhibitory postsynaptic potentials (IPSPs) in olfactory PNs (Waldrop et al. 1987) and abolished odor-induced oscillations (MacLeod and Laurent 1996). The calcium signals were strongly sensitive to GABA: about 2 min after GABA reached the $\mathrm{AL}$, the calcium responses to odors were totally abolished in all animals measured (Fig. 5, $A$ and $C$ ) with a complete recovery after wash-out (5 of 6 animals). Accordant to studies of the moth 


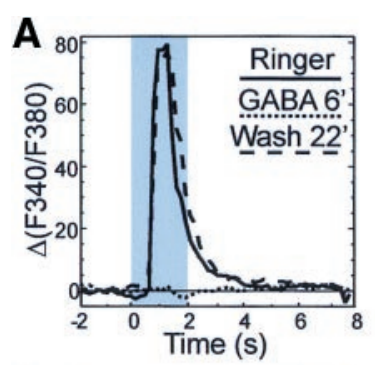

B Spontaneous activity $(n=6)$

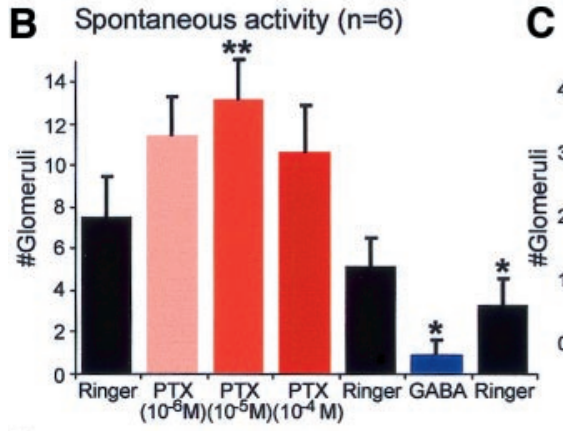

E
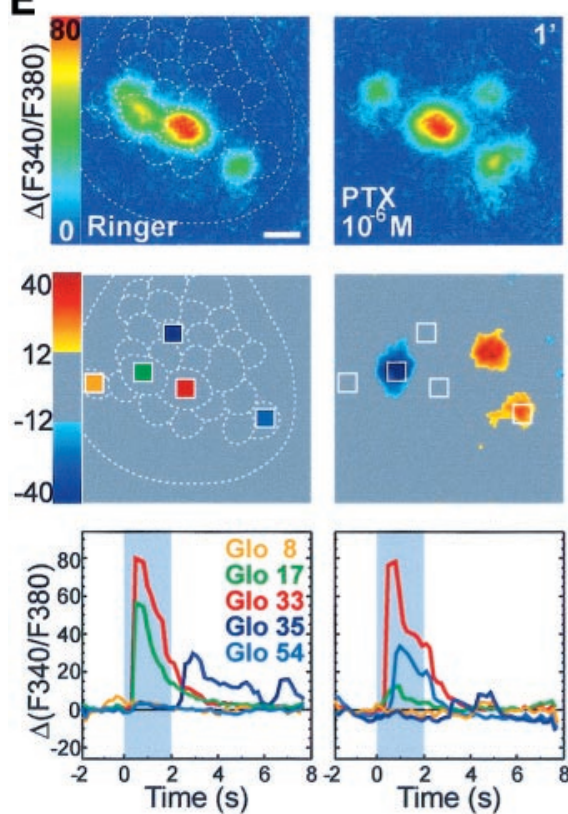
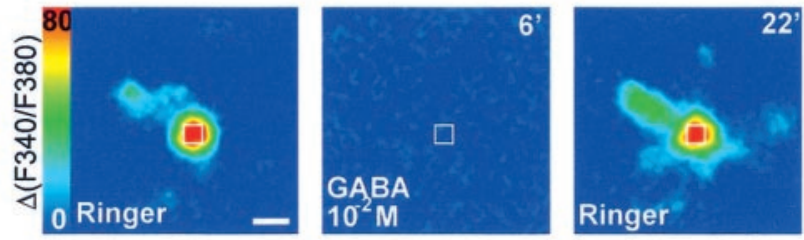

C Responses to nonanol $(\mathrm{n}=6)$
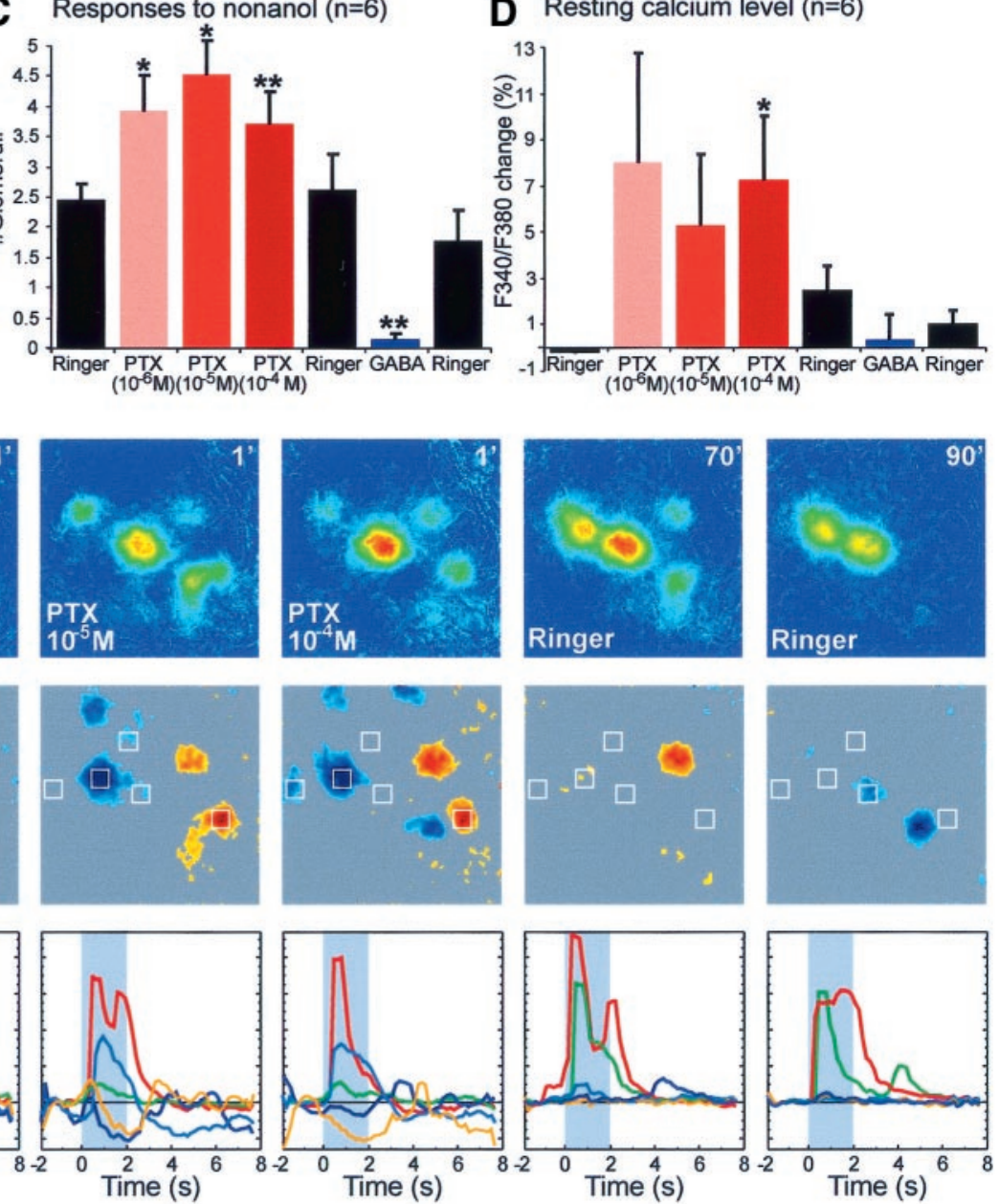

FIG. 5. Application of GABA to the AL totally abolishes the odor-evoked responses of PNs, whereas PTX changes both spatial and temporal components of the signals. A: time traces of glomerulus 33 (left) and false-color coded activity patterns (right) (position of glomerulus 33 corresponds to the square) to nonanol stimulation before, during, and after GABA application. Nonanol stimulation is marked by a shaded area in the time course. $B-D$ : influence of PTX and GABA on the number of spontaneously activated glomeruli, the number of glomeruli responding to nonanol during a measurement of $10 \mathrm{~s}$, and the resting calcium level. Each bar represents mean and SE values of 6 measurements. The arrangement of the different bars from left to right reflects the temporal sequence of the experiment. Asterisks give significant differences to the first Ringer measurement $(* P<0.05$, $* * P<$ 0.01, two-tailed paired $t$-test). E: spatial activity patterns to nonanol (upper row) during a successive increase of the PTX concentration. The glomerular structure has been reconstructed in the first image (compare with Fig. $2 A$ for glomerular identity). By subtracting these activity patterns during or after PTX application from the first pattern (i.e., before PTX), we calculated difference images (middle row), which highlight calcium increases (red) and decreases (blue) due to PTX. These effects almost disappear again in the wash. The bottom row represents the time traces of 5 identified glomeruli, whose positions in the AL are marked by colored boxes in the first image. The numbers at the top right in each frame indicate the time elapsed from the latest perfusion switch. Scale bar, $50 \mu \mathrm{m}$.

Manduca sexta (Christensen et al. 1998; Waldrop et al. 1987), GABA abolished both excitatory and inhibitory responses of PNs. Spontaneous activity also decreased significantly during GABA application (Fig. 5B).
Application of PTX changed both temporal and spatial aspects of the calcium signals in a stereotypical manner. The number of glomeruli that were spontaneously active or that showed excitatory responses to an odor was significantly in- 
creased following PTX treatment (Fig. 5, $B$ and $C$ ). Furthermore, in all glomeruli the resting calcium levels increased with PTX (Fig. 5D), which was apparent in the ratio 340/380 nm. We attribute this calcium increase to the increased spontaneous activity. In our standard protocol, where PTX was applied in steps of increasing concentration $\left(10^{\prime} 10^{-6} \mathrm{M}, 10^{\prime} 10^{-5} \mathrm{M}\right.$, $\left.10^{\prime} 10^{-4} \mathrm{M}\right)$, some PTX effects decreased again at the highest concentration (Fig. 5, $B$ and $C$ ). This is probably due to the prolonged PTX application: in control experiments direct application of $10^{-4}$ PTX led first to a strong increase in spontaneous and odor-evoked activity, which changed after $5 \mathrm{~min}$ of application to a decrease in these effects $(n=3$, data not shown).

PTX-induced changes in the temporal response courses were odor specific and consistent across animals. We found five types of characteristic effects following PTX application. Figure $5 E$ shows an example for nonanol in which the time courses of five glomeruli are shown, one for each observed effect. During PTX application the characteristic diagonal pattern of nonanol changed into a more widespread pattern, because of added glomeruli (e.g., glomerulus 54, cyan line). These added glomeruli are apparent as red in the difference images (middle row in Fig. 5E). Excitatory responses were lengthened by PTX and showed a double peak (e.g., glomerulus 33, red line). Although PTX caused a significant overall increase in the number of activated glomeruli, some glomeruli were reduced in their activity. For example, the response of glomerulus 17 was significantly reduced in the nonanol pattern during PTX application (green time course in Fig. 5E) in all animals measured. A similar reduction appeared in glomeruli 24 and 38 to the odor octanol and hexanol, respectively. We did not observe such an effect for isoamylacetate. Furthermore, PTX treatment increased inhibitory responses or left them unchanged (e.g., glomerulus 8 or 35 in Fig. 5E), which is apparent as blue in the difference images (middle row in Fig. $5 E$ ). Most PTX effects were reversible in the wash but minor changes in the time courses persisted even after long washing periods. Taken together, the five types of effects following PTX application were as follows ("a" to "e" in Fig. 6A): increased response of weakly (a) or strongly (c) activated glomeruli, increased inhibition of inhibited (b) or nonresponding (d) glomeruli (the latter becomes visible due to the increased calcium-resting levels, Fig. 5D) and reduced response of intermediately activated glomeruli (e), which is the opposite effect to (a). These effects were again odor- and glomerulusspecific and consistent across animals $(n=6)$ : for example, glomerulus 33 falls into category a for octanol, into d for isoamylacetate and hexanol and into c for nonanol. Characteristic glomeruli for each effect and odor are given in Fig. 6A. As for the odor responses without pharmacological treatment (Fig. 3 ), we found some variability across animals also for the effects of PTX. Interestingly, effects a and d were less consistently observed for the same glomerulus in different animals, while effects b, c, and e were highly conserved across individuals.

\section{I S C U S S I O N}

In this study we measured the neuronal output of the olfactory glomeruli by optically recording the calcium responses of

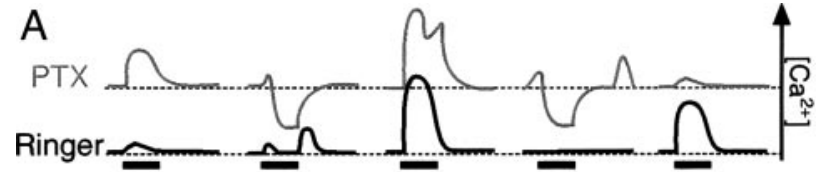

\begin{tabular}{lccccc}
\hline Odours a & b & c & d & e \\
\hline Isoamyl. 35, 38 & 23 & 28,36 & 33 & - \\
\hline Hexanol 35, 39 & 18,24 & 28 & 17,33 & 38 \\
\hline Octanol 28, 33 & 29,35 & 17,52 & 43 & 24 \\
\hline Nonanol 54, 62 & 28,35 & 33 & 8,48 & 17 \\
\hline
\end{tabular}
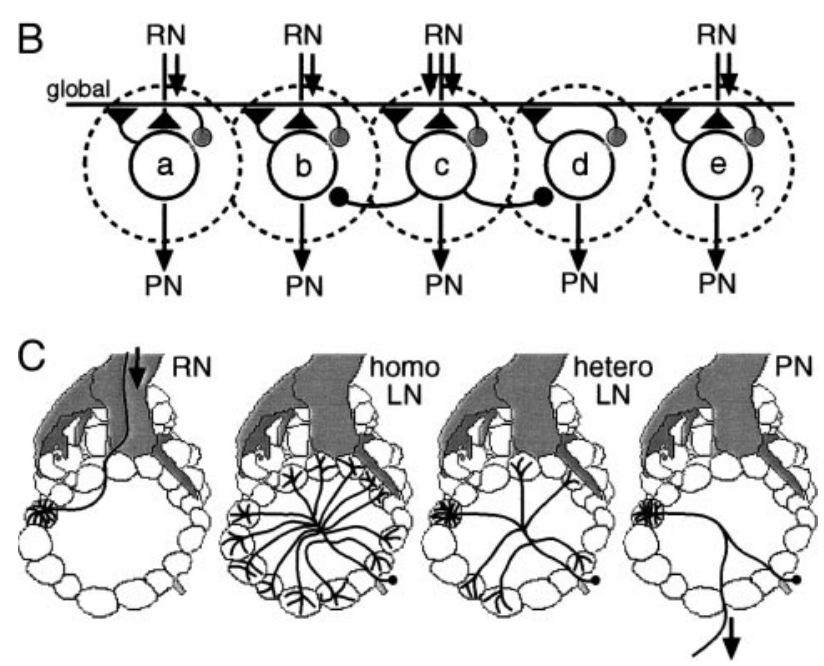

FIG. 6. Two inhibitory networks shape the odor representation of PN responses. A, above: examples of time courses of PN responses to odors; gray: superfusion with PTX; black: Ringer control. PTX leads to increased resting $\left[\mathrm{Ca}^{2+}\right]$ levels and changes the response properties, which can be categorized as 5 types of effects (a-e). Below: examples for identified glomeruli for each category (a-e) for different odors. B: resulting model for the functional connectivity between olfactory glomeruli (labeled according to the categories $\mathrm{a}-\mathrm{e})$ when stimulated with a particular odor. Glomerulus $\mathrm{c}$ gets strong RN input and inhibits other glomeruli with PTX-insensitive synapses (black circles). Glomeruli a, b, and e get weak RN input, d no RN input for this odor. All glomeruli feed into a global, PTX-sensitive inhibitory network (gray circles). Therefore application of PTX leads to an increase of the PN response of weakly activated glomeruli (a) and a prolongation of glomeruli with a strong $\mathrm{RN}$ input (c). The tonic increase in intracellular calcium and spontaneous activity due to PTX leads to the calcium decrease becoming more visible; thus the inhibitory response of inhibited glomeruli is enhanced (b), and inhibitory PN responses during odor stimulation are visible in previously nonresponding glomeruli (d), in particular if they are spontaneously active shortly before the stimulus onset. The reduction of type e glomeruli following PTX application may be due to PN desynchronization. $C$ : cell types present in the honeybee AL. RNs innervate a single glomerulus. Homogeneous LNs innervate diffusely between 30-100 glomeruli. Heterogeneous LNs densely innervate 1 glomerulus and other glomeruli only sparsely. PNs only innervate a single glomerulus. Each glomerulus is innervated by about 3-5 PNs. Multiglomerular PNs and modulatory neurons also innervate the $\mathrm{AL}$ and are not shown here.

selectively stained PNs. These results show that the AL circuitry transforms the input from the RNs into a complex spatio-temporal glomerular pattern, including calcium increases and decreases (Figs. 2, 3, and 4). A similar variety of response properties consisting of both excitatory and inhibitory phases has been shown electrophysiologically in PNs of different insects and in lobsters (Abel 1997; Kanzaki et al. 1989; Sun et al. 1993; Wachowiak and Ache 1998). Electrophysiological recordings combined with calcium imaging of PNs in the honeybee showed that inhibitory responses coincide with a 
calcium decrease in these cells (Kimmerle and Menzel 2000). In motion-sensitive cells of the fly optic system as well as in rat mitral cell dendrites, a perfect correlation between intracellular calcium concentration and membrane potential was observed (Charpak et al. 2001; Single and Borst 1998). The direct input from RNs to PNs is believed to be mediated by nicotinic acetylcholine receptors (Bicker 1999), which are calcium-permeable in cultured honeybee neurons (Goldberg et al. 1999). In locusts the calcium influx through these receptors contributes about $25 \%$ to the total calcium signal (Oertner et al. 1999). Therefore the calcium measured in this study may consist of both a component reflecting the input to the PN and a component proportional to the membrane potential, thus corresponding to the output from the PN. Thus the calcium responses may overestimate the PN spike output to the mushroom bodies. However, in studies combining electro- and opto-physiological recordings of olfactory output neurons in rats (Charpak et al. 2001) and honeybees (Kimmerle and Menzel 2000) a high concordance between both techniques was found, suggesting that calcium responses overestimate the AP-train response only to a limited extent. While imaging techniques allow inspecting the spatial distribution of neural activity, the temporal resolution is substantially reduced as compared with electrophysiological measurements. The time resolution of our experiment $(6 \mathrm{~Hz})$ precludes a precise description of the temporal structure of the PN responses in terms of spike frequency.

Without odor application, the AL showed two types of spontaneous activity, one similar to a diffuse flickering, and another consisting of individual active glomeruli. These two modes of spontaneous activity may reflect the background firing versus the bursting firing of PNs, both of which has been reported in single-cell recordings (Abel 1997; Kanzaki et al. 1989). Alternatively, the stronger activity bouts may correspond to events of simultaneous activity of the three to five PNs innervating each glomerulus. More experiments are needed to address this question.

We found that the odor-evoked response of PNs is determined by both the odor used and the innervated glomerulus. Interestingly, for some odors the excited and inhibited glomeruli clustered together (Fig. 4A). This indicates that in the honeybee inhibitory connections are not limited to neighboring glomeruli. In honeybees, LNs generally innervate individual glomeruli of the spherical AL with branches originating from the coarse neuropil at the center of the AL, so that the wiring length between glomeruli is independent of their physical distance.

The response features of PNs innervating a particular glomerulus are conserved between different individuals (Fig. 3). Still, there is some variability in the glomerular responses when comparing among individuals (Fig. 3) as well as within one animal (data not shown). This variability appears to be higher, compared with our previously published data, where the responses derived mainly from the afferents (Galizia et al. 1999c; Sachse et al. 1999). Part of this is probably due to the animals' individual experiences, since our animals were foragers and therefore not odor-naive in our experiments. Appetitive learning has been shown to influence the glomerular responses (Faber et al. 1999) so that the interindividual differences may reflect different foraging backgrounds. PN responses, representing the output of the AL, are affected more strongly by these plastic changes than RNs. Interestingly, for the most variable odor in our study (octanol), response similarity was correlated with season, but not with beehive. A detailed analysis of this variability is beyond the scope of this paper. Within an experiment, the repeated exposure to odors, even without a reward, can also lead to learning events in the AL, as shown in electrophysiological recordings from locust PNs (Stopfer and Laurent 1999).

Because of the conserved glomerular features among individuals, we assume that the three to five PNs innervating a particular glomerulus have similar response profiles. In contrast to the data presented here, the previously published compound signals are dominated by the afferent input (Galizia et al. 1998). The glomerular pattern of PNs appears more confined than the published compound signal from all AL cells, which is supported by a correlation analysis for each odor pair (Fig. 4, $A$ and $B$ ). We are currently measuring with both staining protocols simultaneously: preliminary results confirm the comparison made here. In addition to the quantitative differences, we also found that the glomerular output can be inhibited by odor stimulation. These differences between AL input and output suggest that the AL network enhances the contrast between different odor representations. These findings agree with electrophysiological recordings of olfactory PNs in vertebrates and invertebrates, which show that the range of odorants eliciting activity in these neurons is typically not as broad as that for sensory neurons (Christensen et al. 1996; Mori and Shepherd 1994). Also, the temporal patterns are sharper than the compound signal, since most of the PN responses are directly stimulus correlated and start or end with stimulus onset or offset (Figs. 2, 3, and 5).

The spatio-temporal sharpening is probably accomplished by the network of inhibitory LNs (Christensen et al. 1993; Sun et al. 1993). A prominent inhibitory neurotransmitter in the honeybee AL is GABA (Schäfer and Bicker 1986). Application of GABA totally abolished the odor representations, whereas PTX led to higher resting calcium levels, more spontaneous activity, more glomeruli responding to an odor (both with excitation and with inhibition), individual glomeruli dropping out of the pattern, and changes in the time courses of the glomerular responses (Fig. 5). In electrophysiological singlecell recordings it has been shown that PTX blocked the oscillatory activity of PNs evoked by odors (MacLeod and Laurent 1996) or by electrical stimulation of the primary afferents (Wehr and Laurent 1999) and that it impairs fine odor discrimination (Stopfer et al. 1997). Oscillations have been observed in a variety of species and are probably controlled by GABAergic LNs (Laurent 1996; MacLeod and Laurent 1996; Wehr and Laurent 1999). They lead to the synchronization of action potentials in PNs as responses to odors (Wehr and Laurent 1996). The significant reduction of some glomerular responses following PTX application (glomeruli of type e in Fig. 6A) may be a consequence of the desynchronization of $\mathrm{PN}$ responses (MacLeod and Laurent 1996). The temporal resolution of our recordings is not sufficient to verify this hypothesis. However, Laurent's group also found that the slow, odor-specific temporal patterns were unaffected by PTX (MacLeod and Laurent 1996; MacLeod et al. 1998; Stopfer et al. 1997), contrary to our observation of substantial changes. This contradiction with our results could be due to the fact that in single-cell recordings 
only a specific subpopulation of PNs is sampled, whereas our recordings reflect the activity in all PNs. Stopfer et al. (1997) interpreted the observed impaired discrimination of related odor stimuli due to PTX as a result of PN desynchronization. On the basis of the new data shown here, this result must be reinterpreted to include both temporal and spatial features. The finding that odor responses of neurons downstream to the PNs were affected by PTX application to the AL (MacLeod et al. 1998) is in line with our results.

Comparing the glomerular PN responses before and after PTX treatment (Figs. 5 and $6 A$ ) allows one to postulate a putative AL network model (Fig. 6B). PTX leads to more glomeruli being active, showing that it affects inhibitory connections. However, strong inhibitory responses still remained, indicating the existence of a second, PTX-insensitive, inhibitory network. Our glomerular connectivity model therefore implies two independent inhibitory networks, in a way akin to what has been proposed for the honeybee's olfactory system based on anatomical data (Linster et al. 1994). The first one is a global inhibitory network which is driven by all glomeruli and affects all glomeruli; it is PTX-sensitive and therefore GABAergic (gray circles in Fig. 6B). The putative respective interneurons have been morphologically described: homogeneous LNs (13\% of LNs) distribute their branches homogeneously over the whole AL and diffusely innervate many glomeruli (Flanagan and Mercer 1989b; Fonta et al. 1993) (Fig. $6 C$ ). Moreover, it has been shown that ca. 750 of the 4000 LNs in the honeybee's AL are GABAergic (Schäfer and Bicker 1986; Witthöft 1967). This PTX-sensitive network effects a global gain control mechanism and could be instrumental for the establishment of oscillations (Laurent 1996). A second inhibitory network is PTX-insensitive and therefore unmasked when the GABAergic system is silenced by PTX application. Its neurons connect specific glomeruli (black circles in Fig. $6 B)$. Figure $6 A$ gives examples for these connections: for example, there is an inhibitory connection from glomerulus 28 to glomeruli 18, 24, 17, and 33 (revealed by stimulating with hexanol), and from glomerulus 33 to glomeruli $28,35,8$, and 48 (revealed by stimulating with nonanol). Note that in these postulated connections the receiving glomerulus is unambiguously identified in our measurements (e.g., glomerulus 24 is clearly inhibited by hexanol). The active glomerulus causing this inhibition (e.g., glomerulus 28 for hexanol) is not unambiguous, because it is likely that other active glomeruli were outside the imaged area. Morphologically, these connections could be accomplished by heterogeneous LNs ( $87 \%$ of LNs), which densely innervate one particular glomerulus and diffusely branch in other glomeruli (Flanagan and Mercer 1989b; Fonta et al. 1993) (Fig. 6C). For this network the inhibitory transmitter remains unclear. A possible candidate would be histamine, which has been shown to be an inhibitory transmitter in the olfactory system of the lobster (Wachowiak and Ache 1997), and for which there is a strong population of immunoreactive local interneurons in the honeybee AL (Bornhauser and Meyer 1997). This network mediates a sort of "lateral" inhibition, where lateral is a functional term and not necessarily related to neighboring glomeruli but rather to specific relationships between response properties in a multidimensional olfactory space (Galizia and Menzel 2000). Indeed, inhibited and inhibiting glomeruli generally have overlapping response profiles in the physiological $\mathrm{AL}$ atlas [http://www .neurobiologie.fu-berlin.de/honeybeeALatlas (Sachse et al. 1999)]. Interestingly, a minimum difference between the glomerular response profiles appears to be required: in the case of aliphatic alcohols, a response range distance of two to four carbon atoms is observed. For example, glomerulus 28, which is maximally activated for hexanol (C6), is inhibited during nonanol (C9) stimulation $(\Delta C=3)$, but not by octanol $(\Delta C=$ $2)$. Thus in terms of olfactory similarity, the inhibitory connections may resemble a "Mexican hat" function. It should be noted, however, that these glomerulus-specific inhibitory connections are not always reciprocal: a glomerulus may inhibit another glomerulus without being inhibited by it (for example, glomerulus 28 inhibits glomerulus 24, but is not inhibited by it).

Our recordings of the spatio-temporal response patterns of the output neurons from the AL in honeybees fill one gap in the analysis of the olfactory code. Together with previous studies of odor-evoked spatial activity patterns of a compound signal and pharmacological experiments, we show that the neural network of the AL optimizes the olfactory code and we propose a wiring model for the neurons involved.

We thank R. Menzel for unlimited support and critical discussions, K. Delaney, G. Laurent, D. Müller, U. Schröter, P. Szyszka, M. Weidert, and M. Wurm for comments on earlier versions of the manuscript, and A. Klawitter for technical assistance. We also thank K. Delaney for teaching us the dye-loading technique.

This work was supported by Volkswagen Grant 1/75-399. NOTE ADDED IN PROOF

We have now experimentally confirmed that histamine acts as an inhibitory transmitter in the honeybee AL. The spontaneous activity as well as the odor responses were strongly reduced during bath application of $10 \mathrm{mM}$ histamine to the honeybee brain. Responses recovered after wash-out $(n=5)$. Further experiments have to be done in order to analyze if histamine is indeed the transmitter of the specific inhibitory network as proposed in this paper, or part of an additional (i.e. third) inhibitory network.

\section{REFERENCES}

ABEL R. Das olfaktorische System der Honigbiene: elektrophysiologische und morphologische Charakterisierung von Antennallobus Neuronen und deren Beteiligung beim olfaktorischen Lernen ( $\mathrm{PhD}$ thesis). Berlin, Germany: Freie Universität, 1997.

ABEl R, RYBAK J, AND MENZEL R. Structure and response patterns of olfactory interneurons in the honeybee, Apis mellifera. J Comp Neurol 437: 363-383, 2001.

Arnold G, Masson C, And Budharugsa S. Comparative study of the antennal lobe and their afferent pathway in the worker bee and the drone (Apis mellifera). Cell Tissue Res 242: 593-605, 1985.

BICKER G. Histochemistry of classical neurotransmitters in antennal lobes and mushroom bodies of the honeybee. Micros Res Tech 45: 174-183, 1999.

Bicker G, KReissl S, AND HofBAuer A. Monoclonal antibody labels olfactory and visual pathways in Drosophila and Apis brains. J Comp Neurol 335: 413-424, 1993.

BORNHAUSER BC AND MEYER EP. Histamine-like immunoreactivity in the visual system and brain of an orthopteran and a hymenopteran insect. Cell Tissue Res 287: 211-221, 1997.

Charpak S, Mertz J, Beaurepaire E, Moreaux L, and Delaney K. Odorevoked calcium signals in dendrites of rat mitral cells. Proc Natl Acad Sci USA 98: 1230-1234, 2001.

Christensen TA, Heinbockel T, And Hildebrand JG. Olfactory information processing in the brain: encoding chemical and temporal features of odors. J Neurobiol 30: 82-91, 1996. 
Christensen TA, WALDROP BR, HARROW ID, AND HildEBRAND JG. Local interneurons and information processing in the olfactory glomeruli of the moth Manduca sexta. J Comp Physiol [A] 173: 385-399, 1993.

Christensen TA, WALdRop BR, AND Hildebrand JG. Multitasking in the olfactory system: context-dependent responses to odors reveal dual GABAregulated coding mechanisms in single olfactory projection neurons. $\mathrm{J} \mathrm{Neu}$ roscience 18: 5999-6008, 1998.

Cinelli AR, Hamilton KA, AND KAUER JS. Salamander olfactory bulb neuronal activity observed by video rate, voltage-sensitive dye imaging. III Spatial and temporal properties of responses evoked by odorant stimulation. J Neurophysiol 73: 2053-2071, 1995.

Clyne PJ, Warr CG, Freeman MR, Lessing D, Kim J, and Carlson JR. A novel family of divergent seven-transmembrane proteins: candidate odorant receptors in Drosophila. Neuron 22: 327-338, 1999.

Delaney K, Davison I, and DenK W. Odour-evoked $\left[\mathrm{Ca}^{2+}\right]$ transients in mitral cell dendrites of frog olfactory glomeruli. Eur J Neurosci 13: 1658 1672, 2001.

EsSLEN J AND KAISSLING K-E. Zahl und Verteilung antennaler Sensillen bei der Honigbiene (Apis mellifera L.). Zoomorphology 83: 227-251, 1976.

Faber T, Joerges J, and Menzel R. Associative learning modifies neural representations of odors in the insect brain. Nature Neurosci 2: 74-78, 1999.

Flanagan D AND MERCER AR. An atlas and 3-D reconstruction of the antennal lobe in the worker honey bee Apis mellifera L. (Hymenoptera: Apidae). Int J Insect Morphol Embryol 18: 145-159, 1989a.

FLANAGAN D AND MERCER AR. Morphology and response characteristics of neurones in the deutocerebrum of the brain in the honeybee Apis mellifera. J Comp Physiol [A] 164: 483-494, 1989b.

FonTA C, Sun XJ, AND Masson C. Morphology and spatial distribution of bee antennal lobe interneurons responsive to odours. Chem Senses 18: 101-119, 1993.

FRIEDRICH RW AND KorschING SI. Combinatorial and chemotopic odorant coding in the zebrafish olfactory bulb visualized by optical imaging. Neuron 18: 737-752, 1997.

FRIEDRICH RW AND KoRSCHING SI. Chemotopic, combinatorial, and noncombinatorial odorant representations in the olfactory bulb revealed using a voltage-sensitive axon tracer. J Neuroscience 18: 9977-9988, 1998.

Galizia CG, Joerges J, Küttner A, FABer T, And Menzel R. A semi-in-vivo preparation for optical recording of the insect brain. J Neurosci Methods 76: 61-69, 1997.

Galizia CG, McIllwrath SL, and Menzel R. A digital three-dimensional atlas of the honeybee antennal lobe based on optical sections acquired by confocal microscopy. Cell Tissue Res 295: 383-394, 1999a.

GALIZIA CG AND MENZEL R. Odour perception in honeybees: coding information in glomerular patterns. Curr Opin Neurobiol 10: 504-510, 2000.

GALizia CG AND MENZEL R. The role of glomeruli in the neural representation of odours: results from optical recording studies. J Insect Physiol 47: 115-130, 2001.

Galizia CG, Menzel R, AND Hölldobler B. Optical imaging of odor-evoked glomerular activity patterns in the antennal lobes of the ant Camponotus rufipes. Naturwissenschaften 86: 533-537, 1999b.

Galizia CG, NäGler K, Hölldobler B, AND Menzel R. Odour coding is bilaterally symmetrical in the antennal lobe of honeybees (Apis mellifera). Eur J Neurosci 10: 2964-2974, 1998.

Galizia CG, Sachse S, and Mustaparta H. Calcium responses to pheromones and plant odours in the antennal lobe of the male and female moth Heliothis virescens. J Comp Physiol [A] 186: 1049-1063, 2000.

Galizia CG, Sachse S, Rappert A, and Menzel R. The glomerular code for odor representation is species specific in the honeybee Apis mellifera. Nature Neurosci 2: 473-478, 1999c.

Gao Q, Yuan B, And Chess A. Convergent projections of Drosophila olfactory neurons to specific glomeruli in the antennal lobe. Nature Neurosci 3: $780-785,2000$

GASCUEL J AND MASSON C. A quantitative ultrastructural study of the honeybee antennal lobe. Tissue Cell 23: 341-355, 1991.

GELPERIN A AND FlORES J. Vital staining from dye-coated microprobes identifies new olfactory interneurons for optical and electrical recording. $J \mathrm{Neu}$ rosci Methods 72: 97-108, 1997.

Goldberg F, Grünewald B, Rosenboom H, And Menzel R. Nicotinic acetylcholine currents of cultured Kenyon cells from the mushroom bodies of the honeybee Apis mellifera. J Physiol (Lond) 514: 759-768, 1999.

HiLdEBRAND JG AND SHEPHERD GM. Mechanisms of olfactory discrimination: converging evidence for common principles across phyla. Annu Rev Neurosci 20: 595-631, 1997.
Joerges J, Küttner A, Galizia CG, And Menzel R. Representation of odours and odour mixtures visualized in the honeybee brain. Nature 387: 285-288, 1997.

KanZaki R, Arbas EA, Strausfeld NJ, and Hildebrand JG. Physiology and morphology of projection neurons in the antennal lobe of the male moth Manduca sexta. J Comp Physiol [A] 165: 427-453, 1989.

KAUER JS, Senseman DM, AND COHEN LB. Odor-elicited activity monitored simultaneously from 124 regions of the salamander olfactory bulb using a voltage-sensitive dye. Brain Res 418: 255-261, 1987.

Kimmerle B AND MenZel R. Spatial and temporal processing of odor information in single antennal lobe neurons of the honeybee. Eur J Neurosci 12: 498, 2000.

LAM Y-W, COHEN LB, Wachowiak M, AND Zochowski MR. Odors elicit three different oscillations in the turtle olfactory bulb. J Neuroscience 20: 749-762, 2000.

Laska M, Galizia CG, Giurfa M, and Menzel R. Olfactory discrimination ability and odor structure-activity relationships in honeybees. Chem Senses 24: 429-438, 1999.

LAURENT G. Dynamical representation of odors by oscillating and evolving neural assemblies. Trends Neurosci 19: 489-496, 1996.

Linster C, Marsan D, Masson C, and Kerszberg M. Advances in Neural Information Processing Systems. San Francisco, CA: Morgan Kaufmann, 1994, p. 527-534.

MACLEOD K, BÄCKER A, AND LAURENT G. Who reads temporal information contained across synchronized and oscillatory spike trains? Nature 395: 693-698, 1998.

MacLeOD K AND LAURENT G. Distinct mechanisms for synchronization and temporal patterning of odor-encoding neural assemblies. Science 274: 976979, 1996.

Margrie TW, Sakmann B, AND URban NN. Action potential propagation in mitral cells lateral dendrites is decremental and controls recurrent and lateral inhibition in the mammalian olfactory bulb. Proc Natl Acad Sci USA 98: 319-324, 2001.

Meister M AND Bonhoeffer T. Tuning and topography in an odor map on the rat olfactory bulb. J Neuroscience 21: 1351-1360, 2001.

MobBs PG. The brain of the honeybee Apis mellifera. I. The connections and spatial organization of the mushroom bodies. Philos Trans $R$ Soc Lond B Biol Sci 298: 309-354, 1982.

Mombaerts P. Molecular biology of odorant receptors in vertebrates. Аппи Rev Neurosci 22: 487-509, 1999.

Mombaerts P, Wang F, Dulac C, Chao SK, Nemes A, Mendelsohn M, EDMONDSON J, AND AXEL R. Visualizing an olfactory sensory map. Cell 87: 675-686, 1996.

MORI K AND SHEPHERD GM. Emerging principles of molecular signal processing by mitral/tufted cells in the olfactory bulb. Sem Cell Biol 5: 65-74, 1994.

OerTner TG, Single S, AND Borst A. Separation of voltage- and ligand-gated calcium influx in locust neurons by optical imaging. Neurosci Lett 274: 95-98, 1999.

RUBIN BD AND KATZ LC. Optical imaging of odorant representations in the mammalian olfactory bulb. Neuron 23: 499-511, 1999.

SACHSE S, RAPPERT A, AND GALIZIA CG. The spatial representation of chemical structures in the antennal lobes of honeybees: steps towards the olfactory code. Eur J Neurosci 11: 3970-3982, 1999.

SCHÄFER S AND BICKER G. Distribution of GABA-like immunoreactivity in the brain of the honeybee. J Comp Neurol 246: 287-300, 1986.

SCHILD D. Principles of odor coding and a neural network for odor discrimination. Biophys J 54: 1001-1011, 1988

SiNGLE S AND Borst A. Dendritic integration and its role in computing image velocity. Science 281: 1848-1850, 1998.

Stopfer M, Bhagavan S, SMith BH, AND LAURENT G. Impaired odour discrimination on desynchronization of odour-encoding neural assemblies. Nature 390: 70-74, 1997.

STOPFER M AND LAURENT G. Short-term memory in olfactory network dynamics. Nature 402: 664-668, 1999.

Sun XJ, Fonta C, AND MAsson C. Odour quality processing by bee antennal lobe interneurons. Chem Senses 18: 355-377, 1993.

SUZUKI H. Antennal movements induced by odor and central projection of the antennal neurones in the honeybee. J Insect Physiol 21: 831-847, 1975.

UChIDA N, TAKAHASHI YK, TANIFUJI M, AND MoRI K. Odor maps in the mammalian olfactory bulb: domain organization and odorant structural features. Nature Neurosci 3: 1035-1043, 2000

Vosshall LB, Amrein H, Morozov PS, Rzhetsky A, And Axel R. A spatial 
map of olfactory receptor expression in the Drosophila antenna. Cell 96: 725-736, 1999.

Vosshall LB, Wong AM, And Axel R. An olfactory sensory map in the fly brain. Cell 102: 147-159, 2000.

WaCHOWIAK M AND ACHE BW. Dual inhibitory pathways mediated by GABAand histaminergic interneurons in the lobster olfactory lobe. J Comp Physiol [A] 180: 357-372, 1997.

WACHOWIAK M AND ACHE BW. Multiple inhibitory pathways shape odorevoked responses in lobster olfactory projection neurons. J Comp Physiol [A] 182: 425-434, 1998.

Waldrop BR, Christensen TA, And Hildebrand JG. GABA-mediated syn- aptic inhibition of projection neurons in the antennal lobe of the sphinx moth Manduca sexta. J Comp Physiol [A] 161: 23-32, 1987.

WEHR M AND LAURENT G. Odour encoding by temporal sequences of firing in oscillating neural assemblies. Nature 384: 162-166, 1996.

WEHR M AND LAURENT G. Relationship between afferent and central temporal patterns in the locust olfactory system. J Neuroscience 19: 381-390, 1999.

WiтtнӧFт W. Absolute Anzahl und Verteilung der Zellen im Hirn der Honigbiene. Z Morphol Tiere 61: 160-184, 1967.

YOKOI M, MORI K, AND NAKANISHI S. Refinement of odor molecule tuning by dendrodendritic synaptic inhibition in the olfactory bulb. Proc Natl Acad Sci USA 92: 3371-3375, 1995. 